\title{
Newer Development in Immunization Practices
}

\author{
A. K. Dutta ${ }^{1} \cdot$ Anju Aggarwal ${ }^{2}$
}

Received: 10 October 2017 / Accepted: 10 October 2017 /Published online: 20 November 2017

(C) Dr. K C Chaudhuri Foundation 2017

Immunization has been the most cost-effective measure to bring about a reduction in childhood mortality and morbidity [1]. Expanded programme on immunization was launched by WHO in 1974 to protect all the children of the world from six diseases $-\mathrm{BCG}$ for tuberculosis, Diptheria-pertussis-tetanus vaccine, oral polio and measles. Hepatitis B was included after $10 \mathrm{y}$ [2]. Since then with increasing availability of vaccines more and more vaccines are been included in the programme. Global agencies are working towards provision of all life saving vaccines to the children of all countries. Vaccines have contributed one of the greatest reductions in under five mortality towards achieving millennium developmental goal in many countries of the world. However the reduction has not been optimum in many developing countries including India due to various reasons.

Immunization has been regarded as one of the major strategy to reach sustainable development goals by 2030 adopted by United nations. "Transforming our world: the 2030 Agenda for Sustainable Development is a set of 17 "Global Goals" with 169 targets among them". The goal is to bring down neonatal mortality to $12 / 1000$ live births and under five mortality to 25 per 1000 live births by 2030 . Hence the main focus is bringing down mortality due to diarrheal diseases and pneumonia, the two main causes of under five mortality [3]. There is specific target to end

A. K. Dutta

drdutta@gmail.com

1 Department of Pediatrics, School of Medical Science and Research, Sharda University, Greater Noida, Uttar Pradesh, India

2 Department of Pediatrics, University College of Medical Sciences and Guru Tegh Bahadur Hospital, New Delhi, India epidemics of tuberculosis, malaria, AIDS and other water borne and communicable diseases [3].

Pneumonia is a major cause of childhood mortality. It accounts for about $25 \%$ of all childhood deaths in India and 15$50 \%$ of them are caused by Streptococcal pneumoniae. Singh A et al. [4] in their article discuss the role of conjugate pneumococcal vaccine (PCV) and its universal use including the types of vaccines to be used. They have analysed various serotypes in the vaccines, efficacy of 10 valent and 13 valent vaccines. WHO has recommended PCV to be included in the immunization schedule of any country with under five mortality $>50 / 1000$ live births in 2007 . PCV 13 is being introduced in India in a phased manner from 2017 under Universal Immunization Programme [5].

Diarrhea remains an important cause of under five mortality. Most of the diarrhea in early childhood is due to rotavirus, especially the dehydrating gastroenteritis. Rotasheild, the first rotavirus vaccine was associated with controversies that it causes intussusception, which was more due to late age of administration of the vaccine. Other controversies as lower immunogenicity in developing countries, strain differences in various formulations have been discussed in depth in the article by Dash $\mathrm{N}$ et al. [6]. Rotavirus vaccine developed from $119 \mathrm{E}$ strain has been indigenously made in India, has been shown to be efficacious and is being introduced in India's National Immunization Schedule in a phased manner. The pentavalent reassortant bovine strain vaccine and monovalent human strain vaccine are effective vaccines. Pertussis is a major cause of mortality and morbidity in young infants with serious complications e.g., encephalitis. Whole-cell pertussis vaccine as a part of DPT vaccine has been recommended since 1974. In 1990's, acellular vaccines were introduced in many developed countries. These were less reactogenic with minor side-effects as fever and local pain. With increasing coverage of vaccines till $5 \mathrm{y}$ of age, pertussis incidence increases in later 
age groups and whenever the coverage decreases, there is surge in pertussis incidence. It was assumed that this could be due to widespread use of acellular vaccines. Controversies regarding the use of acellular vaccines and components of the vaccine have been discussed by Nash D et al. [6]. Hence there is a need for regular boosters and immunization of the unimmunized population. Antenatal pertussis vaccination along with tetanus and diphtheria has been incorporated in routine immunization schedule of all pregnant females in several countries of the world. Needless to say, regular booster doses of pertussis vaccines are necessary to maintain adequate level of immunity in the population.

Malaria, dengue and Japanese encephalitis are mosquito borne diseases which are difficult to prevent as mosquito control measures have not yielded satisfactory results. Vaccines have proven role in prevention of Japanese encephalitis and yellow fever. A live attenuated vaccine, SA-14-14- 2 strain is in use in our National program to prevent Japanese encephalitis. In addition, two more second generation inactivated vero cell indigenous Japanese encephalitis vaccines are available (using SA14-14-2 and Kolar strain respectively) with excellent efficacy. A new vaccine made by chimeric technique using SA14-14-2 and yellow fever is available in the world market. Malaria vaccines against Plasmodium falciparum are in different stages of development. One such vaccine has been tried in Africa with variable results. WHO has initiated a trial with the presently available vaccine in three African countries [7]. However, Dengue vaccine has been licensed for use is countries with seroprevalance of $50-70 \%$, but has only upto $50 \%$ efficacy in $9-45$ y of age. Research on development of effective vaccines against Chikungunya, Zika and west Nile fever are ongoing.

Global eradication strategies for Polio have been eloquently discussed in the article by Bahl et al. The world is almost in the verge of eradication of wild polio virus except in two countries e.g., Afghanistan and Pakistan. The intelligent use of oral polio vaccine along with active surveillance of AFP with virological identification made this program of polio eradication one of the most challenging. However, vaccine derived polio virus (VDPV) is causing threat in several countries of the world where immunity level against polio is poor. Most of these VDPV cases are due to circulating type II vaccine virus. Withdrawal of type II OPV globally and introduction of IPV as an end game strategy is to be practiced now. Future strategies would include withdrawal of all oral polio vaccines and use of killed vaccine with sustained active surveillance to have a polio free world which shall be another milestone in the history of immunization [8].

With increasing number of vaccines included in the immunization schedule, an infant is receiving several painful injections with varieties of adverse reactions related to unsafe injection practices. One of the reason of poor coverage of the vaccine is pain and anxiety due to injections. Therefore alternative routes of vaccine administration and no pain techniques have been developed and are in use in many countries. Garg $\mathrm{N}$ et al. have an interesting review on the use of various other modes of vaccine delivery as oral, edible, sublingual, nasal sprays and nanopatches. Jet injectors and micro needles are also being used in many countries of the world and are very useful for mass vaccination. These methods will require less training of health care workers and may reduce cost of immunization in the long run [9].

Adverse events following vaccination are important to monitor. This can be done with post vaccination surveillance of new vaccines. If a vaccine has adverse events, it is less likely to be acceptable leading to decreased coverage. Various controversies are appearing in the media regarding vaccination and a robust AEFI monitoring program will help to establish whether the events are due to vaccines, programme error or just incidental. Global vaccine action plan expects 10 serious AEFI's for every 100,000 live births, which means about 2600 for India but about 1100 are being reported now. Hence there is urgent need to improve active surveillance of adverse events following vaccination [10].

Immunization still remains as one of the best strategy to reduce the burden of post neonatal infant mortality and morbidity. With the introduction of new vaccines e.g., Pentavalent, Rota, Inactivated Polio and Pneumococcal in National Immunization Schedule, a thorough knowledge on these important vaccines, their proper uses and adverse reactions needs to be understood. Mission Indradhanush (intensified immunization campaign) was launched in 2015 to increase coverage from $65 \%$ to $90 \%$ to all children, free of cost [11]. It aimed at improving coverage in poorly performing districts. It aims to its full immunization coverage by 5 percentage points every year and succeeded in boosting immunization coverage by approx. $6.7 \%$ by vaccinating almost 2.1 crore children [12]. Intensified mission Indradhanush [13] has been launched from October 2017 with the aim to accelerate the full immunization coverage and provide greater focus on urban areas and other pockets of low immunization coverage. According to the plan, states will conduct Intensified Mission Indradhanush drives for 7 working days from 7 th day of every month starting from 7th October 2017 for four consecutive months excluding Sundays, holidays and Routine Immunization days. This will have more mobile staff and more accountability.

With increasing coverage and introduction of newer vaccines, we hope to achieve sustainable developmental goals.

\section{Compliance with Ethical Standards}

Conflict of Interest None.

Source of Funding None. 


\section{References}

1. Available at: https://www.healthypeople.gov/2020/topicsobjectives/topic/maternal-infant-and-child-health. Accessed 6 Oct 2017.

2. Available at: http://www.who.int/immunization/programmes systems/supply_chain/benefits_of_immunization/en/http://www. who.int/immunization/programmes_systems/supply_chain/ benefits_of_immunization/en/. Accessed 6 Oct 2017.

3. Available at: http://www.un.org/sustainabledevelopment/ sustainable-development-goals. Accessed 6 Oct 2017.

4. Singh A, Dutta AK. Pneumococcal vaccines - how many serotypes are enough? Indian J Pediatr. 2017; https://doi.org/10.1007/s12098017-2449-3.

5. New Vaccines in India's Universal Immunization. Available at: http://www.thehindubusinessline.com/economy/policy/fourvaccines-added-to-indiasImmuunisation-programme/ article6173880. Assessed 6 Oct 2017.

6. Dash N, Verma S. Pertussis and rotavirus vaccines - controversies and solutions. Indian J Pediatr. 2017; https://doi.org/10.1007/ s12098-017-2393-2.
7. Aggarwal A, Garg N. Newer vaccines against mosquito-borne diseases. Indian J Pediatr. 2017; https://doi.org/10.1007/s12098-0172383-4.

8. Bahl S, Bhatnagar P, Sutter RW, Roesel S, Zaffran M. Global polio eradication - way ahead. Indian J Pediatr. 2017; https://doi.org/10. 1007/s12098-017-2529-4.

9. Garg N, Aggarwal A. Advances towards painless vaccination and newer modes of vaccine delivery. Indian J Pediatr. 2017; https://doi. org/10.1007/s12098-017-2377-2.

10. Joshi J, Das MK, Polpakara D, Aneja S, Aggarwal MK, Arora NK. Vaccine safety and surveillance for adverse events following immunization (AEFI) in India. Indian J Pediatr. 2017; https://doi.org/10. 1007/s12098-017-2532-9.

11. Ministry of Health and Family Welfare, PIB Press Release Mission Indradhanush Impact. Available at: http://pib.nic.in/newsite/ PrintRelease. aspx?relid=160063. Accessed 25 July 2017.

12. Available at: http://www.thehindubusinessline.com/economy/ policy/four-vaccines-added-to-indiasImmuunisation-programme/ article6173880. Accessed 6 Oct 2017.

13. Health Ministry to launch Intensified Mission Indradhanush. Targets full immunization coverage by 2018. Available at: http:// pib.nic.in/newsite/PrintRelease. aspx?relid=169354. Accessed 7 Oct 2017. 